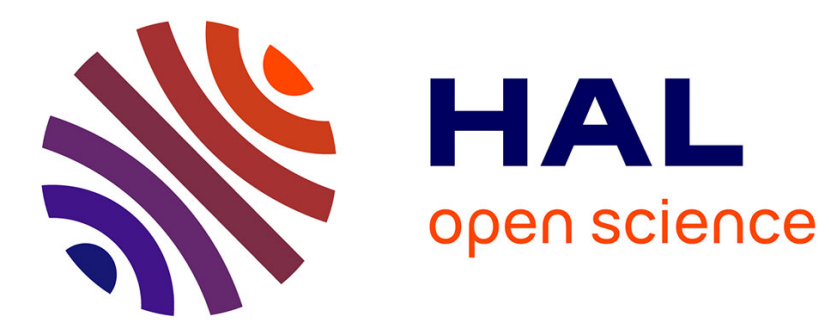

\title{
On Weyl groups in minimal simple groups of finite Morley rank
}

Tuna Altinel, Jeffrey Burdges, Olivier Frécon

\section{To cite this version:}

Tuna Altinel, Jeffrey Burdges, Olivier Frécon. On Weyl groups in minimal simple groups of finite Morley rank. Israel Journal of Mathematics, 2013, 197 (1), pp.377-407. hal-00711484

\section{HAL Id: hal-00711484 \\ https://hal.science/hal-00711484}

Submitted on 25 Jun 2012

HAL is a multi-disciplinary open access archive for the deposit and dissemination of scientific research documents, whether they are published or not. The documents may come from teaching and research institutions in France or abroad, or from public or private research centers.
L'archive ouverte pluridisciplinaire HAL, est destinée au dépôt et à la diffusion de documents scientifiques de niveau recherche, publiés ou non, émanant des établissements d'enseignement et de recherche français ou étrangers, des laboratoires publics ou privés. 


\title{
ON WEYL GROUPS IN MINIMAL SIMPLE GROUPS OF FINITE MORLEY RANK
}

\author{
TUNA ALTINEL, JEFFREY BURDGES, AND OLIVIER FRÉCON
}

\begin{abstract}
We prove that generous non-nilpotent Borel subgroups of connected minimal simple groups of finite Morley rank are self-normalizing. We use this to introduce a uniform approach to the analysis of connected minimal simple groups of finite Morley rank through a case division incorporating four mutually exclusive classes of groups. We use these to analyze Carter subgroups and Weyl groups in connected minimal simple groups of finite Morley rank. Finally, the self-normalization theorem is applied to give a new proof of an important step in the classification of simple groups of finite Morley rank of odd type.
\end{abstract}

\section{INTRODUCTION}

The theory of groups of finite Morley rank has become a well-established branch of the model theory of groups. Most research is centered around the analysis of the infinite simple ones and more precisely the following conjecture independently stated by Gregory Cherlin and Boris Zil'ber.

Algebraicity Conjecture (Cherlin-Zil'ber): An infinite simple group of finite Morley rank, seen as a pure group structure, is a linear algebraic group over an algebraically closed field.

The most important advances in the analysis of the infinite simple groups of finite Morley rank have drawn upon three mathematical resources: the theory of linear algebraic groups over algebraically closed fields, the classification of the finite simple groups, and the analysis of the inherent structure of groups of finite Morley rank using a kind of geometric approach that relying on genericity arguments.

As in the classification of the finite simple groups, the most difficult questions, some of which are still open, have arisen in the analysis of "small" simple groups, in particular, the connected minimal simple groups. These are the ones whose proper, definable, connected subgroups are solvable. Their analysis, that widely varies from one configuration to another, nevertheless permits impressive achievements as soon as the definable subgroup structure is sufficiently rich, a phenomenon reminiscent of the Feit-Thompson theorem in finite group theory. The fundamental articles [CJ04], [Del08], [Fré08] are good examples of this. They deal with torsion elements in a similar way to finite group theory, make use of genericity arguments and approach such notions as the Weyl group in this abstract context.

In this paper, we follow the line of [CJ04], [Del08] and [BD09] while using results and techniques from [Fré08] in order to provide a uniform approach to the analysis of the connected minimal simple groups of finite Morley rank. This approach is

Date: June 11, 2012. 
based on a four-case division whose criteria are the structure of the Weyl group and the intersections of maximal definable connected solvable subgroups (Borel subgroups) and depends on the following central theorem that will be proven in the third section:

Theorem 3.13 Any non-nilpotent generous Borel subgroup B of a minimal connected simple group $G$ is self-normalizing.

It is well-known in algebraic group theory that Borel subgroups are self-normalizing, a fact for which the finite Morley rank analogue is open. Our partial answer uses information on torsion elements (Weyl group) and the generic behaviour of the simple groups in question. This line of reasoning prevails in the proof of the fourcase division that will be accomplished in the fourth section. The efficiency of our case division and of Theorem 3.12 are illustrated in the fifth section where we obtain general structural information on Carter and Borel subgroups of connected minimal simple groups.

The general approach that underlines this article, and that aims at making efficient and conceptual use of the interplay between torsion elements in a group of finite Morley rank and the generic behaviour of the same group, has a striking application to the classification of the infinite simple ones. The final section contains a new and simpler proof of one of the two main steps of the main result of [BCJ07], which is the following theorem:

Theorem 6.1. - If $G$ is a connected minimal simple group of odd type and of Prüfer 2-rank at least two, then $G$ has no strongly embedded subgroup.

The fact that a nontrivial classification theorem has a direct proof as a consequence of the development in this article and its precursors, is convincing evidence that our methods deserve closer attention and are likely to be useful in the work around the Algebraicity Conjecture.

\section{Preliminaries}

We will use a variety of notions and results on groups of finite Morley rank. For our readers' convenience, we include most of them in this section with appropriate references. Nevertheless, we assume some familiarity with the basics of groups of finite Morley rank. An excellent, though somewhat aging, reference is [BN94], while [ABC08] may be of help for more recent developments. The fundamental papers [CJ04], [BCJ07], [Del08] may help focus on some of the insights for this article.

Because of the ordinal character of the Morley rank, a group of finite Morley rank satisfies the descending chain condition on definable subgroups: in a group of finite Morley rank, there is no infinite descending chain of definable subgroups. This property allows one to introduce various notions in the abstract context of groups of finite Morley rank, analogous to geometric aspects of algebraic groups. Thus, the connected component of a group $G$ of finite Morley rank, denoted $G^{\circ}$ and defined as the smallest definable subgroup of finite index, does exist and is the intersection of all definable subgroups of finite index in $G$. A group of finite Morley rank is said to be connected if it is equal to its connected component.

The connected component of a group is an example of a "large" definable set in that it is of the same rank as the ambient group. In general, a definable subset $X$ of $G$ is said to be generic if $\operatorname{RM}(X)=\operatorname{RM}(G)$. Intuitively speaking, a connected group is one where generic subsets intersect generically. 
Dually, if $X$ is an arbitrary subset of a group $G$ of finite Morley rank, then one defines its definable hull, denoted $d(X)$ as the intersection of all definable subgroups of $G$ containing $X$. The descending chain condition implies that the definable hull of a set is well-defined and definable. This is a weak analogue of the Zariski closure in algebraic geometry. The notion of a definable hull allows one to define the connected component of an arbitrary subgroup of the ambient group $G$ : if $X$ is subgroup, then $X^{\circ}$ is defined as $X \cap d(X)^{\circ}$, and $X$ is said to be connected if $X=X^{\circ}$. Various algebraic properties are preserved as one passes to the definable hull:

Fact 2.1. - (Zil'ber) [BN94, Corollary 5.38] If $G$ is a group of finite Morley rank and $H$ a solvable (resp. nilpotent) subgroup of class $n$, then $d(H)$ has the same properties.

A definable set in a group $G$ of finite Morley rank is said to be indecomposable if for any definable subgroup $H \leq G$ whenever cosets of $H$ decompose $X$ into more than one subset, then they decompose it into infinitely many. In particular, an indecomposable subgroup is a connected subgroup. Zil'ber's indecomposability theorem states that indecomposable sets that contain the identity element of the group generate definable connected subgroups. We will use the following corollaries frequently, mostly without mention:

Fact 2.2. - [BN94, Corollary 5.28] Let $G$ be a group of finite Morley rank. Then the subgroup generated by a family of definable connected subgroups of $G$ is definable and the setwise product of finitely many of them.

Fact 2.3. - [BN94, Corollaries 5.29 and 5.32] Let $G$ be a group of finite Morley rank.

(1) Let $H \leq G$ be a definable connected subgroup of $G$ and $X$ an arbitrary subset of $G$. Then the subgroup $[H, X]$ is definable and connected.

(2) Let $H$ be a definable subgroup of $G$. Then the members of the derived $\left(H^{(n)}\right)$ and lower central series $\left(H^{n}\right)$ of $H$ are definable. If $H$ is connected, then so are these subgroups of $H$.

The algebraic structure of an arbitrary group of finite Morley rank naturally exhibits similarities to that of a linear algebraic group. A group of finite Morley rank is built up from definable, minimal subgroups that are abelian:

Fact 2.4. - [Rei] [BN94, Theorem 6.4] In a group of finite Morley rank, a minimal, infinite, definable subgroup $A$ is abelian. Furthermore, either $A$ is divisible or is an elementary abelian p-group for some prime $p$.

This simple and historically old fact is what permits many inductive arguments using Morley rank. The additional structural conclusions in Fact 2.4 are related to the following general structural description of abelian groups of finite Morley rank.

Fact 2.5. - [Mac70Gr, Theorems 1 and 2] [BN94, Theorem 6.7] Let $G$ be an abelian group of finite Morley rank. Then the following hold:

(1) $G=D \oplus C$ where $D$ is a divisible subgroup and $C$ is a subgroup of bounded exponent;

(2) $D \cong \oplus_{p}$ prime $\left(\oplus_{I_{p}} \mathbb{Z}_{p_{\infty}}\right) \oplus \oplus_{I} \mathbb{Q}$ where the index sets $I_{p}$ are finite;

(3) $G=D B$ where $D$ and $B$ are definable characteristic subgroups, $D$ is divisible, $B$ has bounded exponent and $D \cap B$ is finite. The subgroup $D$ is connected. If $G$ is connected, then $B$ can be taken to be connected. 
It easily follows from this detailed description of abelian groups of finite Morley rank that, in general, groups of finite Morley rank enjoy the property of lifting torsion from definable quotients. More precisely, if $G$ is a group of finite Morley rank, $H \leq G$ a definable subgroup of $G$ and $g \in G$ such that $g^{n} \in H$ for some $n \in \mathbb{N}^{*}$, where $n$ is assumed to be the order of $g$ in $d(g) / d(g) \cap H$ and is a $\pi$-number with $\pi$ a set of prime numbers, then there exists $g^{\prime} \in g H \cap d(g)$ such that $g^{\prime}$ is again a $\pi$-element. Here, a $\pi$-number is a natural number whose prime divisors belong to $\pi$, and a $\pi$-element is an element whose order is a $\pi$-number. This torsion-lifting property will be of crucial use in the analysis of Weyl groups in Section 3 and will be used without mention.

Fact 2.5 has a nilpotent analogue:

Fact 2.6. - [Nes91, Theorem 2] [BN94, Theorem 6.8 and Corollary 6.12] Let $G$ be a nilpotent group of finite Morley rank. Then $G$ is the central product $B * D$ where $D$ and $B$ are definable characteristic subgroups of $G, D$ is divisible, $B$ has bounded exponent. The torsion elements of $D$ are central in $G$.

We will need the following elementary property of nilpotent groups:

Fact 2.7. - [BN94, Lemma 6.3] Let $G$ be a nilpotent group of finite Morley rank and $H$ a definable subgroup of infinite index in $G$. Then $N_{G}(H) / H$ is infinite.

The structural properties of the solvable groups of finite Morley rank vary considerably from those of the nilpotent ones. Mostly, the differences are best measured by field structures that are definable in solvable non-nilpotent groups of finite Morley rank. Solvable groups of finite Morley rank tend to have a "definably linear" character as illustrated by the following fundamental fact:

Fact 2.8. - (Zil'ber) [BN94, Theorem 9.1] Let $G$ be an infinite, connected, solvable, non-nilpotent group of finite Morley rank. Then there exist a field $K$ and definable connected sections $U$ and $T$ of $G^{\prime}$ and $G / G^{\prime}$ respectively such that $U \cong(K,+)$, and $T$ embeds in $\left(K^{\times}, \cdot\right)$. Moreover, these mappings are definable in the pure group $G$, and each element of $K$ is the sum of a bounded number of elements of $T$. In particular, $K$ is definable in $G$ and hence of finite Morley rank.

We recall an old but fundamental theorem about fields of finite Morley rank:

Fact 2.9. - [Mac71Fi, Theorem 1] [BN94, Theorem 8.1] A field definable in a structure of finite Morley rank is either finite or algebraically closed.

The ability to define an algebraically closed field in a connected solvable eventually culminates in the following result that generalizes a well-known property of connected solvable algebraic groups.

Fact 2.10. - [BN94, Corollary 9.9] Let $G$ be a connected solvable group of finite Morley rank. Then $G^{\prime}$ is nilpotent.

The Fitting subgroup of a group of finite Morley rank $G$, denoted $F(G)$, is defined to be the maximal, definable, normal, nilpotent subgroup of $G$. By works of Belegradek and Nesin, this definition is equivalent to the one in finite group theory: the subgroup generated by all normal, nilpotent subgroups. Nesin has also proven:

Fact 2.11. - [BN94, Theorem 9.21] Let $G$ be a connected solvable group of finite Morley rank. Then $G / F(G)^{\circ}$, thus also $G / F(G)$, are divisible abelian groups. 
A Borel subgroup of a group of finite Morley rank is a maximal, definable, connected, solvable subgroup.

For each prime $p$, a Sylow p-subgroup of any group $G$ is defined to be a maximal locally finite $p$-subgroup.

\section{Fact 2.12.}

(1) [BN94, Theorem 6.19] For any prime number $p$, a locally finite p-subgroup of a group of finite Morley rank is nilpotent-by-finite.

(2) [BN94, Proposition 6.18 and Corollary 6.20] If $P$ is a nilpotent-by-finite $p$-subgroup of a group of finite Morley rank, then $P^{\circ}=B * T$ is the central product of a definable, connected, subgroup $B$ of bounded exponent and a divisible abelian p-group. In particular, $P^{\circ}$ is nilpotent.

For each prime $p$, a nilpotent definable connected $p$-group of finite Morley rank is said to be $p$-unipotent if it has bounded exponent while a $p$-torus is a divisible abelian $p$-group.

In general, a $p$-torus is not definable. It is the direct sum of finitely many copies of $\mathbb{Z}_{p^{\infty}}$, the Sylow $p$-subgroup of the multiplicative group of the complex numbers. In particular, the $p$-elements of order at most $p$ form a finite elementary abelian $p$-group whose rank is called the Prüfer $p$-rank of the torus in question.

The choice of terminology, "unipotent" and "torus", is not coincidental. Fact 2.12 (2) shows that the Sylow $p$-subgroups of a group of finite Morley rank have similarities with those of algebraic groups. These are of bounded exponent when the characteristic of the underlying field is $p$, and divisible abelian when this characteristic is different from $p$. In the notation of Fact $2.12(2)$, this case division corresponds to $T=1$ or $B=1$ respectively when the Sylow $p$-subgroup in question is non-trivial.

A similar case division for the prime 2 has played a major role in developing a strategy to attack parts of the Cherlin-Zil'ber conjecture. In this vein, a group of finite Morley rank is said to be of even type if its Sylow 2-subgroups are infinite of bounded exponent $(B \neq 1, T=1)$, of odd type if its Sylow 2-subgroups are infinite and their connected components are divisible $(B=1, T \neq 1)$, of mixed type if $B \neq 1$ and $T \neq 1$ and of degenerate type if they are finite.

The main result of [ABC08] states that a simple group of finite Morley rank that contains a non-trivial unipotent 2-subgroup is an algebraic group over an algebraically closed field of characteristic 2 . In this article, we will use this result and refer to it as the classification of simple groups of even type. Despite spectacular advances for groups of odd type, no such extensive conclusion has been achieved. In the degenerate type, it has been shown in [BBC07] that a connected group of finite Morley rank of degenerate type has no involutions:

Fact 2.13. - [BBC07, Theorems 1 and 3] Let $G$ be a connected group of finite Morley rank whose maximal p-subgroups are finite. Then $G$ contains no elements of order $p$.

The following generalization of a well-known semisimple torsion property of algebraic groups was proven following a similar line of ideas.

Fact 2.14. - [BC08b, Theorem 3] Let $G$ be a connected group of finite Morley rank, $\pi$ a set of primes, and a any $\pi$-element of $G$ such that $C_{G}(a)^{\circ}$ does not contain a non-trivial $\pi$-unipotent subgroup. Then a belongs to any maximal $\pi$-torus of $C_{G}(a)$. 
The generic element of a group of finite Morley rank is strongly related to the structure of its tori. A divisible abelian group $G$ of finite Morley rank is said to be: a decent torus if $G=d(T)$ for $T$ its (divisible) torsion subgroup; a pseudo-torus if no definable quotient of $G$ is definably isomorphic to $K_{+}$for an interpretable field $K$. It follows from elementary torsion properties of groups of finite Morley rank that a decent torus is a pseudo-torus. The following remark based on important work of Wagner on bad fields of non zero characteristic was the first evidence of the relevance of these notions of tori.

Fact 2.15. - [AC04, Lemma 3.11] Let $F$ be a field of finite Morley rank and nonzero characteristic. Then $F^{\times}$is a good torus.

A good torus is a stronger version of a decent torus in that the defining property of a decent torus is assumed to be hereditary.

Using the geometry of groups of finite Morley rank provided by genericity arguments that we will outline later in this section, Cherlin and later the third author obtained the following conjugacy results.

Fact 2.16. -

(1) [Che05, Extended nongenericity] In a group of finite Morley rank, maximal decent tori are conjugate.

(2) [Fré09, Theorem 1.7] In a group of finite Morley rank, maximal pseudo-tori are conjugate.

By Fact 2.16 (1), the Prüfer $p$-rank of a group of finite Morley rank can be defined as the Prüfer $p$-rank of a maximal $p$-torus.

We quote the facts about decent and pseudo-tori that we will need. They help illustrate that these more general notions of tori, introduced to investigate more efficiently the structure of groups of finite Morley rank, share crucial properties of tori in algebraic groups, and thus of illuminating what aspects of a notion of algebraic torus influence the structure of algebraic groups.

Fact 2.17.

(1) [Fré06b, Lemma 3.1] Let $G$ be a group of finite Morley rank, $N$ be a normal definable subgroup of $G$, and $T$ be a maximal decent torus of $G$. Then $T N / N$ is a maximal decent torus of $G / N$ and every maximal decent torus of $G / N$ has this form.

(2) [Fré09, Corollary 2.9] Let $G$ be a connected group of finite Morley rank. Then the maximal pseudo-torus of $F(G)$ is central in $G$.

(3) [AB09, Theorem 1] Let $T$ be a decent torus of a connected group G of finite Morley rank. Then $C_{G}(T)$ is connected.

(4) [Fré09, Corollary 2.12] Let $T$ be a pseudo-torus of a connected group $G$ of finite Morley rank. Then $C_{G}(T)$ is connected and generous in $G$, and $N_{G}\left(C_{G}(T)\right)^{\circ}=C_{G}(T)$.

Before moving from tori to the unipotent side, we introduce a notion that is related to both sides and thus fundamental to the understanding of groups of finite Morley rank: Carter subgroups. In groups of finite Morley rank, Carter subgroups are defined as being the definable connected nilpotent subgroups of finite index in their normalizers.

In a reductive algebraic group, Carter subgroups correspond to maximal tori. Hence, the notion of Carter subgroup offers a possibility to approach properties 
of algebraic tori in a purely group-theoretic form. Carter subgroups have strong ties with the geometry of groups of finite Morley rank stemming from genericity arguments. We will review some of these connections later in this section around Fact 2.26.

We will need the following results on Carter subgroups:

Fact 2.18. - Let $G$ be a group of finite Morley rank.

(1) [FJ05], [FJ08, Theorem 3.11] G has a Carter subgroup.

(2) [Fré09, Corollary 2.10] Each pseudo-torus is contained in a Carter subgroup of $G$.

(3) [Wag94, Theorem 29] If $G$ is solvable, its Carter subgroups are conjugate.

(4) [Fré08, Theorem 1.2] If $G$ is a minimal connected simple group, its Carter subgroups are conjugate.

(5) [Fré00a, Théorèmes 1.1 and 1.2] If $G$ is connected and solvable, any subgroup of $G$ containing a Carter subgroup of $G$ is definable, connected and selfnormalizing.

The notions of reduced rank and $U_{0, r}$-groups were introduced by the second author in order to carry out an analogue of local analysis in the theory of the finite simple groups. In a similar vein, a theory of Sylow $U_{0, r}$-subgroups was developed. The notion of homogeneity was introduced by the third author in his refinement of the unipotence analysis. The following definition lists the notions that will be used in this article:

Definition 2.19. - [Bur04], [Fré06a], [Bur06]

- An abelian connected group A of finite Morley rank is indecomposable if it is not the sum of two proper definable subgroups. If $A$ is indecomposable and $A \neq 1$, then $A$ has a unique maximal proper definable connected subgroup $J(A)$, and if $A=1$, let $J(1)=1$.

- The reduced rank of any abelian indecomposable group A of finite Morley rank is $\bar{r}(A)=r k(A / J(A))$.

- For any group $G$ of finite Morley rank and any positive integer $r$, we define

$$
\begin{array}{r}
U_{0, r}(G)=\langle A \leq G| A \text { is indecomposable definable abelian, } \\
\bar{r}(A)=r, A / J(A) \text { is torsion-free }\rangle .
\end{array}
$$

- A group $G$ of finite Morley rank is said to be a $U_{0, r}$-group whenever $G=$ $U_{0, r}(G)$, and to be homogeneous if each definable connected subgroup of $G$ is a $U_{0, r}$-subgroup.

- The radical $U_{0}(G)$ is defined as follows. Set $\bar{r}_{0}(G)=\max \left\{r \mid U_{0, r}(G) \neq 1\right\}$ and set $U_{0}(G)=U_{0, \bar{r}_{0}(G)}(G)$.

- In any group $G$ of finite Morley rank, a Sylow $U_{0, r}$-subgroup is a maximal, definable, nilpotent $U_{0, r}$-subgroup.

Fact 2.20.

(1) [Bur04, Theorem 2.16] Let $H$ be a connected solvable group of finite Morley rank. Then $U_{0}(H) \leq F(H)$.

(2) [Bur06, Corollary 3.5] Let $G$ be a nilpotent group of finite Morley rank. Then $G=D * B$ is a central product of definable characteristic subgroups $D, B$ where $D$ is divisible and $B$ has bounded exponent. The latter group is connected if and only if $G$ is connected. 
Let $T$ be the torsion part of $D$. Then we have decompositions of $D$ and $B$ as follows.

$$
\begin{gathered}
D=d(T) * U_{0,1}(G) * U_{0,2}(G) \ldots \\
B=U_{2}(G) \oplus U_{3}(G) \oplus \ldots
\end{gathered}
$$

For a prime $p, U_{p}(G)$ is the largest normal $p$-unipotent subgroup of $G$.

The work of the third author shows that the theory of unipotence is much better behaved when the unipotent groups in question are homogeneous in the sense of Definition 2.19. The following fact is efficient in finding homogeneous groups.

Fact 2.21. - [Fré06a, Theorem 4.11] Let $G$ be a connected group of finite Morley rank. Assume that $G$ acts definably by conjugation on $H$, a nilpotent $U_{0, r}$-group . Then $[G, H]$ is a homogeneous $U_{0, r}$-group.

As was mentioned earlier, unipotence results are key to developing local analysis in connected minimal simple groups of finite Morley rank. The following major ingredients of local analysis will be used in the sequel.

Fact 2.22. - Let $G$ be a minimal connected simple group. Let $B_{1}, B_{2}$ be two distinct Borel subgroups.

(1) [Bur07, Lemma 2.1] If we have $U_{p_{1}}\left(B_{1}\right) \neq 1$ and $U_{p_{2}}\left(B_{2}\right) \neq 1$, then $F\left(B_{1}\right) \cap$ $F\left(B_{2}\right)=1$.

(2) [Bur07, Lemmas 3.5 and 3.6] Assume that $B_{1}$ and $B_{2}$ are two distinct Borel subgroups such that $\left(B_{1} \cap B_{2}\right)^{\circ}$ is maximal with respect to containment, and that $F\left(B_{1}\right) \cap F\left(B_{2}\right) \neq 1$. If $\bar{r}_{0}\left(B_{1}\right) \geq \bar{r}_{0}\left(B_{2}\right)$, then $\bar{r}_{0}\left(B_{1}\right)>\bar{r}_{0}(H)$ and $\bar{r}_{0}\left(B_{2}\right)=\bar{r}_{0}(H)$, where $H=\left(B_{1} \cap B_{2}\right)^{\circ}$.

(3) [Bur07, Proposition 4.1] Any definable connected nilpotent subgroup of $B_{1} \cap$ $B_{2}$ is abelian.

We close this section with a short overview of the geometric theory of groups of finite Morley rank. Finite groups are discrete structures, and their structure is frequently analyzed using counting arguments that tend to yield conjugacy theorems. On the other hand, density arguments tend to prevail in the realm of algebraic groups, and occasionally result in conjugacy results. In the theory of groups of finite Morley rank one has recourse to both techniques, and occasionally profits from the interplay between the finite and the infinite. A nice example of such an interplay is provided by Weyl groups, the main theme of this article.

The geometric analysis of groups of finite Morley rank mostly involves genericity arguments. A definable subset $X$ of a group $G$ of finite Morley rank is said to be generous in $G$ (or shortly, "generous" in case the ambient group is clear) if the union of its conjugates is generic in $G$. This notion was introduced and studied in [Jal06]. The following were proven in [Jal06]:

Fact 2.23. - Let $G$ be a group of finite Morley rank and $H$ a definable, generous subgroup of $G$.

(1) [Jal06, Lemma 2.2] The subgroup $H$ is of finite index in $N_{G}(H)$.

(2) [Jal06, Lemma 2.3] If $X$ is a definable subset of $H$ that is generous in $G$, then $X$ is generous in $H$.

(3) [Jal06, Lemma 2.4] If $H$ is connected and $X$ is a definable generic subset of $H$, then $X$ is generous in $G$. 
The first point in the above fact, despite its simple nature and proof, illustrates the relationship between generic sets, Weyl groups and the presence of torsion in a group of finite Morley rank.

The following caracterization of generosity is due to Cherlin who was inspired by [Jal06]. It is a relatively simple but efficient illustration of the geometry of genericity arguments.

Fact 2.24. - [ABC08, Lemma IV 1.25][Jal06, Section 3.2] Let $G$ be a connected group of finite Morley rank and $H$ definable, connected, and almost self-normalizing subgroup of $G$. Let $\mathcal{F}$ be the family of all conjugates of $H$ in $G$. Then the following are equivalent.

(1) $H$ is generous in $G$.

(2) The definable set

$$
H_{0}=\{h \in H:\{X \in \mathcal{F}: h \in X\} \text { is finite }\}
$$

is generic in $H$.

(3) The definable set

$$
G_{0}=\left\{x \in \bigcup_{g \in G} H^{g}:\{X \in \mathcal{F}: x \in X\} \text { is finite }\right\}
$$

is generic in $G$.

As has been mentioned, there is a close connection between conjugacy and genericity although this does not in general necessitate an implication in either direction. The conjugacy results on decent and pseudo-tori go via genericity arguments. In [Jal06], Jaligot proved the conjugacy of generous Carter subgroups of groups of finite Morley rank. The most general conjugacy result for Carter subgroups that does not depend on the generosity hypothesis was proven in [Fré08] for $K^{*}$-groups, that is the groups of finite Morley rank all of whose simple definable proper sections are algebraic. Fact 2.18 (4) is the important particular case that deals with the minimal connected simple groups and that we will need in this paper. In general, it remains a major open problem whether one can remove the $K^{*}$ hypothesis.

Fact 2.25. - [Jal06, Part of Corollary 3.8] Le G be a group of finite Morley rank and $C$ a Carter subgroup of $G$. Then the following are equivalent:

(1) $C$ is generous in $G$.

(2) $C$ is generically disjoint from its conjugates.

A definable set $X$ is generically disjoint from its conjugates if $\operatorname{RM}\left(X \backslash \bigcup_{g \in G \backslash \operatorname{Stab}_{G}(X)}\right)=$ $\operatorname{RM}(X)$.

Fact 2.26. - Let $G$ be a group of finite Morley rank. Then the following hold:

(1) [Jal06, Theorem 3.1] All generous Carter subgroups are conjugate;

(2) [FJ08, Theorem 3.11] [CJ04, Lemma 3.5] if $G$ is solvable, its Carter subgroups are generically disjoint and generous.

The following observation about minimal connected simple groups illustrates the connection between genericity, Carter subgroups and torsion elements.

Fact 2.27. - [AB09, Proposition 3.6] Let $G$ be a minimal connected simple group. If $G$ has non-trivial torsion, then $G$ has a generous Carter subgroup. 


\section{The Weyl group of a minimal Connected simple group of finite MORLEY RANK}

There are several definitions proposed for Weyl groups in groups of finite Morley rank: in a group $G$ of finite Morley rank, one can consider $N_{G}(C) / C$ where $C$ is a Carter subgroup of $G$, or $N_{G}(T) / C_{G}(T)$ where $T$ is a maximal decent or pseudo-torus. In simple algebraic groups, these possibilities yield natural, uniquely defined, robust notions that are also equivalent. This equivalence is not known in an arbitrary simple group of finite Morley rank.

Fact 2.18 (1) or the definition of a decent or pseudo-torus show that one can define a notion of Weyl group in a group of finite Morley rank. Nevertheless, the definition using Carter subgroups cannot yield a uniquely defined notion as long as it is not known whether in general Carter subgroups are conjugate in groups of finite Morley rank, an open problem. Thanks to Fact 2.16, this problem is overcome in the case of the definitions involving tori, and we define the Weyl group $W(G)$ of a group $G$ of finite Morley rank to be $N_{G}(T) / C_{G}(T)$ where $T$ is any maximal decent torus of $G$.

Our first target in the present section is to verify that in a minimal connected simple group, the definition of a Weyl group that we have adopted is in fact equivalent to the other above-mentioned possibilities. This will be done mainly in Proposition 3.2 and followed up in Corollaries 3.4 and 3.5.

The second target of this section involves the proof of the central result of this paper. We will use our development of a robust notion of Weyl group to analyze another well-known property of simple algebraic groups ([Hum81, Theorem 23.1]) in the context of groups of finite Morley rank, namely the self-normalization of Borel subgroups. This problem is open even in the context of minimal connected simple groups of finite Morley rank. We will prove in Theorem 3.12 that the property holds in a minimal connected simple group under additional hypotheses.

An important ingredient of our arguments is the conjugacy of Carter subgroups in minimal connected simple groups (Fact $2.18(4)$ ). We will also need the following fact which can be regarded as a very weak form of self-normalization:

Fact 3.1. - [AB09, Lemma 4.3] If $B$ is a Borel subgroup of a minimal connected simple group $G$ such that $U_{p}(B) \neq 1$ for some prime number $p$, then $p$ does not divide $\left[N_{G}(B): B\right]$.

Proposition 3.2. - Let $G$ be a minimal connected simple group, and let $C$ be a Carter subgroup of $G$. Then the Weyl group $W(G)$ of $G$ is isomorphic to $N_{G}(C) / C$.

Proof - Let $T$ be a maximal decent torus of $G$. Then $T$ is contained in a Carter subgroup of $G$ (Fact $2.18(2)$ ) and, by the conjugacy of Carter subgroups (Fact $2.18(4)$ ), we may assume $T \leq C$. By Fact 2.6, we have $C \leq C_{G}(T)^{\circ}$. If $T$ is non-trivial, then $C_{G}(T)$ is a connected solvable subgroup of $G$ by Fact 2.17 (3). In particular $C$ is self-normalizing in $C_{G}(T)$ (Fact $2.18(5)$ ), and Fact $2.18(3)$ and a Frattini Argument yield $N_{G}(T)=C_{G}(T) N_{G}(C)$. Hence we obtain

$$
N_{G}(C) / C \simeq N_{G}(T) / C_{G}(T) \simeq W(G) .
$$

Suppose now that $T=1$, that is, $G$ contains no non-trivial decent torus (Fact $2.16(1))$

We assume toward a contradiction that $N_{G}(C) / C$ is non-trivial. Then there is a prime $p$ dividing the order of $N_{G}(C) / C$. Let $S$ be a Sylow $p$-subgroup of $G$. 
Since there exists no non-trivial decent torus in $G$, Fact $2.12(2)$ implies that $S^{\circ}$ is a $p$-unipotent subgroup of $G$. Moreover, it is non-trivial by Fact 2.13 . Let $B$ be a Borel subgroup containing $S^{\circ}$. Then we have $S^{\circ} \leq U_{p}(B)$ and Fact $2.22(1)$ shows that $B$ is the unique Borel subgroup containing $S^{\circ}$. In particular, $S$ normalizes $B$ and $U_{p}(B)$, and we obtain $S^{\circ}=U_{p}(B)$ by maximality of $S$. Thus we have $N_{G}(B)=N_{G}\left(S^{\circ}\right)$.

Let $D$ be a Carter subgroup of $B$ (Fact 2.18 (1)). If some $B$-minimal section $\bar{A}$ of $S^{\circ}$ is not centralized by $B$, then $B / C_{B}(\bar{A})$ is definably isomorphic to a definable subgroup of $K^{\times}$for a definable algebraically closed field $K$ of characteristic $p$ (Fact $2.8)$, and Fact 2.15 shows that $B / C_{B}(\bar{A})$ is a decent torus. Then there is a nontrivial decent torus in $B$ by Fact 2.17 (1), contradicting our hypothesis. Thus $D$ centralizes each $B$-minimal section of $S^{\circ}$, and this implies $S^{\circ} \leq D$ since $N_{B}(D)^{\circ}=$ $D$. Now $N_{G}(D)$ normalizes $S^{\circ}=U_{p}(D)$ and we have $N_{G}(D) \leq N_{G}\left(S^{\circ}\right)=N_{G}(B)$. In particular $D$ is a Carter subgroup of $G$ and we may assume $D=C$ (Fact $2.18(4)$ ). By the conjugacy of Carter subgroups in $B$ (Fact $2.18(3)$ ), the self-normalization of $C$ in $B$ (Fact $2.18(5)$ ), and a Frattini Argument, we obtain $N_{G}(B)=B N_{G}(C)$ and

$$
N_{G}(C) / C=N_{G}(C) /\left(N_{G}(C) \cap B\right) \simeq N_{G}(B) / B .
$$

This implies that $p$ divides the order of $N_{G}(B) / B$, contradicting Fact 3.1.

This result has the following consequence, which is similar to a classical result for algebraic groups [Hum81, Exercise 6 p.142].

Corollary 3.3. - If $C$ is a Carter subgroup of a minimal connected simple group $G$, then $C$ is a maximal nilpotent subgroup.

Proof - Let $D$ be a nilpotent subgroup of $G$ containing $C$. By Fact 2.1, we may assume $D$ is definable. Since $N_{G}(C) / C$ is finite, Fact 2.7 implies that $C$ has finite index in $D$, and thus $C=D^{\circ}$. Let $T$ be the maximal decent torus of $C$. Then $T$ is maximal in $G$ by Fact 2.18 (2) and (4). Thus, if $T=1$, then Proposition 3.2 gives $N_{G}(C)=C$ and $D=C$. On the other hand, if $T \neq 1$, then $C_{G}(T)$ is a connected solvable group by Fact 2.17 (3), and it contains $D$ (Fact 2.6). Now, by Fact $2.18(5)$, we obtain $D \leq N_{C_{G}(T)}(C)=C$, proving the maximality of $C$.

Corollary 3.4. - Let $G$ be a minimal connected simple group, and let $S$ be a nontrivial p-torus for a prime $p$. Then $N_{G}(S) / C_{G}(S)$ is isomorphic to a subgroup of $W(G)$. Moreover, if $S$ is maximal, then we have $N_{G}(S) / C_{G}(S) \simeq W(G)$.

Proof - By Fact $2.18(2), S$ is contained in a Carter subgroup $C$ of $G$. By Fact 2.6, we have $C \leq C_{G}(S)^{\circ}$. Since $S$ is non-trivial, then $C_{G}(S)$ is a connected solvable group Fact 2.17 (3), and $C$ is self-normalizing in $C_{G}(S)$ (Fact $2.18(5)$ ). Now a Frattini Argument yields $N_{G}(S)=C_{G}(S) N_{N_{G}(S)}(C)$, and $N_{G}(S) / C_{G}(S)$ is isomorphic to a subgroup of $N_{G}(C) / C \simeq W(G)$.

Moreover, if $S$ is maximal, then $S$ is characteristic in $C$, and we have $N_{G}(C)=$ $N_{N_{G}(S)}(C)$. Hence we obtain $W(G) \simeq N_{G}(C) / C \simeq N_{G}(S) / C_{G}(S)$.

Corollary 3.5. - Let $G$ be a minimal connected simple group, and let $T$ be a maximal pseudo-torus of $G$. Then $W(G)$ is isomorphic to $N_{G}(T) / C_{G}(T)$.

Proof - When $T \neq 1$, we proceed as in the first paragraph of the proof of Proposition 3.2. By Facts 2.18 (2) and $2.17(2), T$ is a central subgroup of a Carter subgroup $C$ of $G$. If $T$ is non-trivial, then Fact 2.17 (4) and Fact 2.18 (3) and 
(5) provide $N_{G}(C) / C \simeq N_{G}(T) / C_{G}(T)$. It then follows from Proposition 3.2 that $W(G) \simeq N_{G}(T) / C_{G}(T)$.

Suppose now that $T=1$. Note that in general, every decent torus is a pseudotorus. Thus by Fact $2.16(2)$, every maximal decent torus in $G$ is trivial as well. It follows that both $N_{G}(T) / C_{G}(T)$ and $W(G)$ are trivial.

Now, we move on to the problem of self-normalization of Borel subgroups. We will need several results from [Del08] and [BD09], that we will present in a form more suitable for our needs.

We begin by reformulating a large portion of the main theorem of [Del08]. In doing so, we will use the conjugacy of Carter subgroups of minimal connected simple groups and some of the preceding results. For the sake of completeness, we detail how these new ingredients intervene in the proof together with Fact 2.17 (3).

Fact 3.6. - (Particular case of [Del08, Théorème-Synthèse]) Let $G$ be a minimal simple group of odd type. Then $G$ satisfies one of the following four conditions:

- $G \simeq \mathrm{PSL}_{2}(K)$ for an algebraically closed field $K$ of characteristic $p \neq 2$;

- $|W(G)|=1$, and the Prüfer 2-rank of $G$ is one;

- $|W(G)|=2$, the Prüfer 2-rank of $G$ is one, and $G$ has an abelian Borel subgroup $C$;

- $|W(G)|=3$, the Prüfer 2-rank of $G$ is two, and the Carter subgroups of $G$ are not Borel subgroups.

Proof - First we note that, by Fact 2.17 (3) and Corollary 3.4, our definition of a Weyl group is equivalent to the one of [Del08, Théorème-Synthèse].

If the Prüfer 2-rank of $G$ is one and if $W(G)$ is non-trivial, then, by [Del08, Théorème-Synthèse] (1), either we have $G \simeq \mathrm{PSL}_{2}(K)$ for an algebraically closed field $K$ of characteristic $p \neq 2$, or $G$ has an abelian Borel subgroup $C$ such that $N_{G}(C)=C \rtimes\langle i\rangle$ for an involution $i$ inverting $C$. In particular, we have $|W(G)|=2$.

Hence we may assume that the Prüfer 2-rank of $G$ is at least two. By [Del08, Théorème-Synthèse], the Prüfer 2-rank of $G$ is two and we have $|W(G)|=3$. Let $S$ be a Sylow 2-subgroup of $G$. Since $G$ is of odd type, $S^{\circ}$ is a non-trivial 2-torus, it is contained in a Carter subgroup $C$ of $G$ (Fact $2.18(2)$ ), and $S^{\circ}$ is central in $C$ (Fact 2.6). Since [Del08, Théorème-Synthèse] (2) says that $C_{G}\left(S^{\circ}\right)^{\circ}$ is abelian and divisible, we obtain $C=C_{G}\left(S^{\circ}\right)^{\circ}$. Moreover, [Del08, Théorème-Synthèse] (2) says that $C$ is not a Borel subgroup, so no Carter subgroup of $G$ is a Borel subgroup by Fact 2.18 (4).

As for [BD09], the second part of the following fact, rather than the cyclicity of the Weyl group, will be needed in the sequel.

Fact 3.7. - [BD09, Theorem 4.1] Let $G$ be a minimal connected simple group, $T$ a maximal decent torus of $G$, and $\tau$ the set of primes $p$ such that $\mathbb{Z}_{p^{\infty}}$ embeds into $T$. Then $W(G)$ is cyclic, and has an isomorphic lifting to $G$. Moreover, no element of $\tau$ divides $|W(G)|$, except possibly 2.

The results of [BD09, §3] do not need that the group $G$ be degenerate, but just that $|W(G)|$ be odd. This increases their relevance for us in conjunction with results from $[\mathrm{BC} 08 \mathrm{~b}]$. In particular, the following fact holds.

Fact 3.8. - $[\mathrm{BD} 09, \S 3][\mathrm{BC} 08 \mathrm{~b}, \S 5]$ Let $G$ be a minimal connected simple group, $T$ a maximal decent torus of $G, \tau$ the set of primes $p$ such that $\mathbb{Z}_{p \infty}$ embeds into $T$, 
and $\tau^{\prime}$ its complement. If $W(G)$ is non-trivial and of odd order, then the following conditions hold:

(1) [BC08b, Corollary 5.3] the minimal prime divisor of $|W(G)|$ does not belong to $\tau$;

(2) [BD09, Corollary 3.8] $C_{G}(T)$ is a Carter subgroup of $G$;

(3) [BD09, Theorem 3.1, Proposition 3.10] if $B_{T}$ is a Borel subgroup containing $C_{G}(T)$, and if either there is a $\tau^{\prime}$-element normalizing $T$ and $B_{T}$, or there is a prime $q$ such that the Prüfer $q$-rank of $T$ is $\geq 3$, then $C_{G}(T)=B_{T}$.

It is worth noting that point (3) of Fact 3.8 is true even when $W(G)$ is of even order.

The following lemma does not necessitate minimal simplicity.

Lemma 3.9. - Let $B_{1}$ and $B_{2}$ be two generous Borel subgroups of a group $G$ of finite Morley rank. Then there exists $g \in G$ such that $B_{1} \cap B_{2}^{g}$ contains a generous Carter subgroup of $G$.

Proof - Let $C_{i}$ be a Carter subgroup of $B_{i}$ for $i=1,2$. Then $C_{i}$ is generous in $B_{i}$ by Fact $2.26(2)$, and $C_{i}$ is generous in $G$ by Fact 2.23 (3). This implies that $C_{i}$ has finite index in its normalizer in $G$ (Fact $2.23(1)$ ), therefore $C_{i}$ is a Carter subgroup of $G$. Now $C_{1}$ and $C_{2}$ are conjugate (Fact 2.26 (1)), and there exists $g \in G$ such that $C_{2}^{g}=C_{1} \leq B_{1} \cap B_{2}^{g}$.

Lemma 3.10. - Let $G$ be a minimal connected simple group with a nilpotent Borel subgroup $B$. Then $B$ is a Carter subgroup of $G$, and the generous Borel subgroups of $G$ are conjugate with $B$, and they are generically disjoint.

Proof - By Fact 2.4, $B$ is non-trivial, so $N_{G}(B)^{\circ}$ is solvable and $B$ is a Carter subgroup of $G$.

Let $B_{0}$ be a generous Borel subgroup of $G$. Then $B_{0}$ contains a Carter subgroup $C$ of $G$ (Lemma 3.9), and Fact 2.18 (4) implies that $B$ is conjugate to $C$, thus to $B_{0}$. The generic disjointness follows from Fact 2.25.

Now, we prove the self-normalization theorem. In the end of the proof, as noted there as well, we could quote Fact 3.7 to finish quickly. Nevertheless, we will give a slightly longer but direct argument for two reasons. The first is that the quick ending is in fact longer in that it uses the full force of [BD09], which we do not need here. The second and more important reason is that in Section 6, as explained after Fact 6.4 , it will be crucial to have a self-normalization argument that deals with the special case when the Weyl group is of odd order in order to avoid referring to [Del08]. We achieve this goal by reducing the proof of Theorem 3.12, at the end of its first paragraph, to the case where the Weyl group of the ambient group is of odd order, and then avoid using any argument that necessitates the use of [Del08], in particular the full force of [BD09]. The care about the hypothesis in Fact 3.8, that assumes the Weyl group to be of odd order, is also part of these efforts.

The direct approach will use the following classical result:

Fact 3.11. - [ABC08, Lemmas IV.10.16 and IV.10.18] Let T be a p-torus of Prüfer p-rank 1 or 2, where $p$ is a prime, and $\alpha$ an automorphism of $T$ of order $p$, with a finite centralizer in $T$. Then $p \in\{2,3\}$.

Theorem 3.12. - Any non-nilpotent generous Borel subgroup $B$ of a minimal connected simple group $G$ is self-normalizing. 
Proof - We consider a non-nilpotent generous Borel subgroup $B$ of a minimal connected simple group $G$. If $|W(G)|$ is even, then Fact 2.13 and the classification of simple groups of even type shows that either $B$ is self-normalizing, or $G$ is of odd type. In the second case, Fact 3.6 and Lemma 3.10 imply that $G \simeq \operatorname{PSL}_{2}(K)$ for an algebraically closed field $K$, so $B$ is self-normalizing. Hence we may assume that $|W(G)|$ is odd.

We assume toward a contradiction that $B$ is not self-normalizing. By Lemma 3.9, $B$ contains a (generous) Carter subgroup $C$ of $G$. By Fact 2.18 (3) and a Frattini argument, we have $N_{G}(B)=B N_{N_{G}(B)}(C)$, so $C$ is not self-normalizing, and the Weyl group of $G$ is non-trivial (Proposition 3.2). Moreover $\left|N_{G}(B) / B\right|$ divides $|W(G)|$. Let $T$ be the maximal decent torus of $C$. By Facts 2.18 (4) and 3.8 (2), $T$ is a maximal decent torus of $G$ and we have $C=C_{G}(T)$.

Let $p$ be a prime divisor of $\left|N_{G}(B) / B\right|$. Since we have $N_{G}(B)=B N_{N_{G}(B)}(C)$, there is a $p$-element $w$ in $N_{N_{G}(B)}(C) \backslash B$ such that $w^{p} \in B$. In particular we have $w \in N_{G}(T) \backslash C_{G}(T)$ and, by Fact 3.8 (3), the maximal $p$-torus $R$ of $T$ is non-trivial of Prüfer $p$-rank 1 or 2.

At this point, Fact 3.7 allows to finish the proof since it yields a contradiction. As was explained above, we will not do this and give a more direct final argument.

Let $R_{0}=C_{R}(w)^{\circ}$. It is a $p$-torus and we have $w \in C_{G}\left(R_{0}\right)$. Moreover, we have $C \leq C_{G}(R) \leq C_{G}\left(R_{0}\right)$ and $C_{G}\left(R_{0}\right)$ is connected by Fact 2.17 (3). Thus, if $R_{0}$ is non-trivial, then $C$ is a Carter subgroup of the connected solvable subgroup $C_{G}\left(R_{0}\right)$, and Fact $2.18(5)$ yields $w \in N_{C_{G}\left(R_{0}\right)}(C)=C$, contradicting our choice of $w$. Hence $R_{0}$ is trivial and, since Fact $2.18(5)$ implies $w^{p} \in N_{B}(C)=C=C_{G}(T) \leq C_{G}(R)$, the element $w$ induces an automorphism $\varphi$ of order $p$ of $R$ such that $C_{R}(\varphi)$ is finite. Then, since we have $p \neq 2$, Fact 3.11 implies $p=3$. But $|W(G)|$ is odd, so $p$ is the smallest prime divisor of $|W(G)|$, contradicting Fact 3.8 (1). The proof is over.

We use local analytic methods to refine our understanding of the relationships between Carter subgroups of minimal connected simple groups, the Borel subgroups containing these and the Weyl group of the ambient group. The conclusions in the remainder of this section will not be used in the following sections.

Proposition 3.13. - Let $H$ be a subgroup of a minimal connected simple group $G$. If $H$ contains a Carter subgroup $C$ of $G$, then $H$ is definable, and either it is contained in $N_{G}(C)$, or it is connected and self-normalizing.

Proof - We may assume that $H$ is proper in $G$. First we show that $H$ is definable. The subgroup $H_{0}$ generated by the conjugates of $C$ contained in $H$ is definable and connected by Fact 2.2. Since $H$ has been assumed to be proper and $G$ is a minimal simple group, $H_{0}$ is solvable. By conjugacy of Carter subgroups in $H_{0}$ (Fact $2.18(3)$ ) and a Frattini argument, we obtain $H=H_{0} N_{H}(C)$. Thus $H_{0}$ has finite index in $H$, so $H$ is definable. Note also that $H^{\circ}=H_{0}$.

From this point on, we will assume that $H$ is not contained in $N_{G}(C)$. We note that this assumption implies $H^{\circ} \nless \leq N_{G}(C)$. Thus, if the result holds for connected groups, then $H^{\circ}$ is self-normalizing, and we have $H=H^{\circ}$. Hence we may assume that $H$ is connected. In particular, $H$ is solvable.

We assume that $H$ is a maximal connected counterexample to the proposition. Since the conjugacy of Carter subgroups in $H$ (Fact $2.18(3))$ and a Frattini argument yield $N_{G}(H)=H N_{N_{G}(H)}(C)$, the quotient group $N_{G}(H) / H$ is isomorphic to 
a subgroup of $W(G)$ by Proposition 3.2, and $W(G)$ is non-trivial. By Facts 2.27 and $2.18(4), C$ is generous in $G$.

We consider a Borel subgroup $B$ containing $H$. By Fact 2.18 (5), the subgroup $B$ does not contain $N_{G}(H)$. Since $B$ contains $H>C$, it is non-nilpotent and generous in $G$. So it follows from Theorem 3.12 that $B$ is self-normalizing, and we obtain $H<B$.

We will denote by $U$ and $V$ respectively, either $U_{p}(B)$ and $U_{p}(H)$ in case $U_{p}(H)$ is non-trivial for some prime number $p$, or $U_{0}(B)$ and $U_{0, \bar{r}_{0}(B)}(H)$. In particular, we have $B=N_{G}(U)$. If $H$ contains $U$, then $N_{G}(H)$ normalizes $U$, contradicting $B=N_{G}(U)$, hence $H$ does not contain $U$. In particular we obtain $V<N_{U}(V)^{\circ}$ and $H<N_{G}(V)^{\circ}$. Now the maximality of $H$ forces $N_{G}(V)=N_{G}(V)^{\circ}$. But, if $V$ is non-trivial, then Fact $2.18(5)$ shows that $N_{G}(V)^{\circ}$ does not contain $N_{G}(H)$, and since $N_{G}(H)$ normalizes $V$, this contradicts $N_{G}(V)=N_{G}(V)^{\circ}$. Hence $V$ is trivial, and $H^{\prime}$ centralizes $U$ by Facts 2.10 and $2.20(2)$. Thus we have $N_{G}\left(H^{\prime}\right)^{\circ}>H$, and the maximality of $H$ provides $N_{G}\left(H^{\prime}\right)=N_{G}\left(H^{\prime}\right)^{\circ}$. On the other hand, $H^{\prime}$ is non-trivial since $H>C$ is non-nilpotent. Then Fact $2.18(5)$ implies $N_{G}(H) \nless$ $N_{G}\left(H^{\prime}\right)^{\circ}$, contradicting that $N_{G}(H)$ normalizes $H^{\prime}$ and that $N_{G}\left(H^{\prime}\right)=N_{G}\left(H^{\prime}\right)^{\circ}$. This finishes our proof.

The following strengthens Fact 3.8 (2):

Corollary 3.14. - Let $G$ be a minimal connected simple group with a non-trivial Weyl group, and let $T$ be a non-trivial maximal $p$-torus of $G$ for a prime $p$. Then $C_{G}(T)$ is a Carter subgroup of $G$.

Proof - Since $W(G)$ and $T$ are non-trivial, $C_{G}(T)$ is not self-normalizing by Corollary 3.4. But Facts $2.18(2)$ and 2.6 show that $C_{G}(T)$ contains a Carter subgroup $C$ of $G$, so $C_{G}(T)$ is contained in $N_{G}(C)$ by Proposition 3.13. Since $C_{G}(T)$ is connected by Fact 2.17 (3), we obtain the result.

Furthermore, when $W(G)$ is of odd order, we may slightly refine this result. Since this corollary will not be needed later, we will allow ourselves to use Fact 3.7. We emphasize that this application will not use the full force of this fact, namely the cyclicity of the Weyl group.

Corollary 3.15. - Let $G$ be a minimal connected simple group, let $T$ be a nontrivial maximal $p$-torus of $G$ for a prime $p$, and let $V=\left\langle x \in T \mid x^{p}=1\right\rangle$. If $W(G)$ is non-trivial and of odd order, then $C_{G}(V)$ is a Carter subgroup of $G$.

Proof - By Corollary 3.14, $C=C_{G}(T)$ is a Carter subgroup of $G$. Then $T$ is a characteristic subgroup of $C$, and since $V$ is characteristic in $T$, we find $N_{G}(C) \leq N_{G}(T) \leq N_{G}(V)$.

By Fact $2.18(5)$, the subgroup $C$ is self-normalizing in $N_{G}(V)^{\circ}$. Since $W(G)$ is non-trivial, Proposition 3.2 shows that $N_{G}(V)^{\circ}$ does not contain $N_{G}(C)$. In particular, by the previous paragraph, $N_{G}(V)$ is not connected, and Proposition 3.13 implies $N_{G}(V)=N_{G}(C)$.

At this stage, we have

$$
C=C_{G}(T) \leq C_{G}(V) \leq N_{G}(V)=N_{G}(C)=N_{G}(T),
$$

so $C_{G}(V) / C_{G}(T)$ is a subgroup of $N_{G}(C) / C$. Now Proposition 3.2 implies that $C_{G}(V) / C_{G}(T)$ is finite and of odd order, and Fact 3.7 shows that $p$ does not divide its order. 
It is well-known that any finite $p^{\prime}$-group of automorphisms of a finite abelian $p$-group $P$, which acts trivially on the set of the elements of order $p$ of $P$, is trivial (see for example, [Gor68, Theorem 2.4, p. 178]). Thus, $C_{G}(V)$ centralizes the finite subgroups $\left\langle x \in T \mid x^{p^{n}}=1\right\rangle$ of $T$ for each $n \in \mathbb{N}$. It follows that $C_{G}(V)$ centralizes $T$, and we obtain $C_{G}(V)=C_{G}(T)=C$ as desired.

\section{Tetrachotomy theOREM}

In Theorem 4.1, we will carry out a fine analysis of minimal connected simple groups according to two criteria. The first criterion is the existence of a non-trivial Weyl group. This criterion is motivated by the important role played by Weyl groups in minimal connected simple groups of finite Morley rank. When the Weyl group is non-trivial, it determines many structural aspects of the ambient group as was exemplified in the classification of simple groups of even type or in [Del08]. On the other hand, when it is trivial, the ambient group has very high chances of being torsion-free, and the arguments tend to use the geometry of $G$ as in [Fré08].

Our second criterion is the size of the intersections of Borel subgroups. It was already noticed in [Jal01] that the lack of intersection between Borel subgroups makes it very difficult to analyze minimal connected simple groups. "Large" intersections, like in the classification of the finite simples groups, allow a certain kind of local analysis. We have set the following concrete criterion in order to measure whether a minimal connected simple group admits largely intersecting Borel subgroups: the absence of a Borel subgroup generically disjoint from its conjugates other than itself.

The following table introduces the four types of groups that emerge from these two criteria:

\begin{tabular}{|l|l|l|l|}
\cline { 3 - 3 } \multicolumn{2}{c|}{} & \multicolumn{2}{|l|}{$\begin{array}{l}\text { A Borel subgroup generically dis- } \\
\text { joint from its conjugates }\end{array}$} \\
\cline { 3 - 4 } \multicolumn{2}{c|}{} & exists & does not exist \\
\hline \multirow{2}{*}{ Weyl group } & trivial & $(1)$ & $(2)$ \\
\cline { 2 - 4 } & non-trivial & $(3)$ & $(4)$ \\
\hline
\end{tabular}

Theorem 4.1. - (Tetrachotomy theorem) Any minimal connected simple group $G$ satisfies exactly one of the following four conditions:

- $G$ is of type (1), its Carter subgroups are generous and any generous Borel subgroup is generically disjoint from its conjugates;

- $G$ is of type (2), it is torsion-free and it has neither a generous Carter subgroup, nor a generous Borel subgroup;

- $G$ is of type (3), its generous Borel subgroups are nilpotent: they are the Carter subgroups;

- $G$ is of type (4), its Carter subgroups are generous, and there is no nilpotent Borel subgroup.

In the sequel, by "type (i)" we will mean one of the four types caracterized in Theorem 4.1 .

Remark 4.2.

- Bad groups [BN94, Chapter 13], and more generally full Frobenius groups [Jal01], are examples of groups of type (1). The existence of any of these groups is a well-known open problem. 
- The minimal connected simple groups with a nongenerous Carter subgroup are of type (2) and are analyzed in [Fré08].

- The group $\mathrm{PSL}_{2}(K)$ for an algebraically closed field $K$, is of type (4).

- By Fact 2.13, the classification of simple groups of even type, and Theorem 4.1, a non-algebraic minimal connected simple group with involutions is of odd type and not of type (2). But the existence of a minimal connected simple group with involutions and either of type (1), or of type (3), or not algebraic and of type (4), is an open problem (compare Fact 3.6 and Theorem 4.1). Furthermore, the description of three pathological configurations by Deloro in [Del08] (see Fact 3.6) corresponds to the groups of type (1), (3) and (4) respectively.

The following technical lemma is general and will provide the main argument for the first parf of the proof of Theorem 4.1.

Lemma 4.3. - Let $G$ be a group of finite Morley rank, $K \leq H$ definable, connected subgroups of $G$ such that $N_{G}(K) \leq N_{G}(H), K$ is generically disjoint from its conjugates in $G$ and almost self-normalizing. Then $H$ is almost self-normalizing and generically disjoint from its conjugates in $G$.

Proof - By Fact 2.24, $K$ is generous in $G$. Thus $H$ is generous in $G$. By Fact 2.23 (1), $H$ is almost self-normalizing in $G$, and by Fact 2.23 (2), $K$ is generous in $H$. We define $\mathcal{K}_{G}=\left\{K^{g} \mid g \notin N_{G}(H)\right\}$ and $\mathcal{K}_{H}=\left\{K^{g} \mid g \in N_{G}(H)\right\}$. Note that these two sets form a partitioning of the set of all the conjugates of $K$ in $G$. Since $N_{G}(K) \leq N_{G}(H), K \backslash \bigcup \mathcal{K}_{G} \supseteq K \backslash\left(\bigcup_{g \notin N_{G}(K)} K^{g}\right)$. Thus, $K \backslash \bigcup \mathcal{K}_{G}$ is generic in $K$.

Now, suppose towards a contradiction that $H$ is not generically disjoint from its conjugates in $G$, equivalently the set $H \cap\left(\bigcup_{g \notin N_{G}(H)} H^{g}\right)$ is generic in $H$. Then, since $\bigcup_{g \notin N_{G}(H)} H^{g}$ is invariant under the action of $H$ by conjugation, the generosity of $K$ in $H$ implies that of $K \cap \bigcup_{g \notin N_{G}(H)} H^{g}$ in $H$. Thus $K \cap \bigcup_{g \notin N_{G}(H)} H^{g}$ is generic in $K$ by Fact $2.23(2)$.

We set $X=H \backslash \bigcup \mathcal{K}_{H}$. Then $H=X \cup\left(\bigcup \mathcal{K}_{H}\right)$. So,

$$
\begin{aligned}
\bigcup_{g \notin N_{G}(H)} H^{g} & =\bigcup_{g \notin N_{G}(H)}\left(X \cup \bigcup \mathcal{K}_{H}\right)^{g} \\
& =\bigcup_{g \notin N_{G}(H)} X^{g} \cup \bigcup_{g \notin N_{G}(H)}\left(\cup \mathcal{K}_{H}\right)^{g} \\
& =\bigcup_{g \notin N_{G}(H)} X^{g} \cup \bigcup \mathcal{K}_{G},
\end{aligned}
$$

and

$$
K \cap \bigcup_{g \notin N_{G}(H)} H^{g}=\left(K \cap \bigcup_{g \notin N_{G}(H)} X^{g}\right) \cup\left(K \cap \bigcup \mathcal{K}_{G}\right) .
$$

But $K \backslash \cup \mathcal{K}_{G}$ is generic in $K$, therefore $K \cap\left(\bigcup \mathcal{K}_{G}\right)$ is not generic in $K$. Since by the previous paragraph $K \cap \bigcup_{g \notin N_{G}(H)} H^{g}$ is generic in $K, K \cap \bigcup_{g \notin N_{G}(H)} X^{g}$ is generic in $K$ as well. Consequently, since $K$ is generous in $G$, so is $X$ by Fact 2.23 (3). Since the set $X$ is generous in $H$ by Fact 2.23 (2) and is invariant under the action of $H$ by conjugation, it is generic in $H$. This contradicts the generosity of $K$ in $H$ since $\mathcal{K}_{H} \supseteq\left\{K^{g} \mid g \in H\right\}$. The conlusion follows.

Proof of Theorem 4.1 - First we note that, by Fact 2.18 (4), either all the Carter subgroups of $G$ are generous, or $G$ has no generous Carter subgroup. We will divide our discussion into two cases:

Case I: $W(G)=1$, equivalently, $G$ is of type (1) or (2). 
We first prove that if $B$ is a generous Borel subgroup of $G$, then it is generically disjoint from its conjugates in $G$. The core of the argument is provided by Lemma 4.3. We will apply it in the special case of a pair $C \leq B$ where $B$ is a generous Borel subgroup of $G$ and $C$ is a Carter subgroup of $B$. For such a pair, Facts 2.26 (2) and 2.23 (3) imply that $C$ is generous in $B$ and then in $G$. It then follows from Fact 2.23 (1) that $C$ is a Carter subgroup of $G$. Since $C$ is generous, by Fact 2.25 it is generically disjoint from its conjugates in $G$. Finally, the assumption of Case I and Proposition 3.2 imply that $N_{G}(C)=C \leq N_{G}(B)$. In fact, one can rapidly deduce that both $B$ and $C$ are self-normalizing. After this preparation, Lemma 4.3 yields that $B$ is also generically disjoint from its conjugates in $G$.

If $G$ is of type (2), the preceding paragraph shows that $G$ has no generous Borel subgroup. In particular, $G$ has no generous Carter subgroup, and $G$ is torsion-free by Fact 2.27 .

If $G$ is of type (1), it remains to prove that $G$ has a generous Carter subgroup. But a Borel subgroup $B$ of $G$, generically disjoint from its conjugates, is generous in $G$ by Fact 2.24. Hence $G$ has a generous Carter subgroup by Lemma 3.9.

Case II: $G$ is of type (3) or (4).

In this case, $G$ is not torsion-free, and its Carter subgroups are generous by Facts 2.27 and $2.18(4)$.

If $G$ is of type (3), we show that its Carter subgroups are generous Borel subgroups. Let $B$ be a Borel subgroup of $G$ generically disjoint from its conjugates. Then $B$ is generous in $G$ by Fact 2.24 , and by Lemma $3.9, B$ contains a generous Carter subgroup $C$ of $G$. Since $W(G)$ is non-trivial, there exists $w \in N_{G}(C) \backslash C$ (Proposition 3.2), and Fact 2.18 (5) implies $w \notin B$. If $B \neq C$, then Theorem 3.12 gives $B^{w} \neq B$ and $C \leq B^{w}$. This implies that $B$ is generically covered by its conjugates (Fact $2.26(2)$ ), and contradicts our choice of $B$. Hence we have $B=C$, and any Carter subgroup of $G$ is a generous Borel subgroup. Conversely, any generous Borel subgroup of $G$ is a Carter subgroup of $G$ by Lemma 3.10.

If $G$ is of type (4), then $G$ has no nilpotent Borel subgroup by Lemma 3.10. Indeed, a nilpotent Borel subgroup of $G$ would be a Carter subgroup of $G$, and as we have already noted, in types (3) and (4), the Carter subgroups of $G$ are generous, thus generically disjoint from their conjugates. This last conclusion contradicts the assumption (4).

\section{Structure of Carter subgroups in simple groups of type (4)}

As Remark 4.2 suggests, minimal connected simple algebraic groups over algebraically closed fields are of type (4). Thus, one expects simple groups of type (4) to have properties close to those of algebraic groups. The following result provides evidence in this direction by refining the structural description of a Carter subgroup of a group of type (4).

Theorem 5.1. - Let $G$ be a minimal connected simple group of type (4). Then there is an interpretable field $K$ such that each Carter subgroup definably embeds in $K^{\times} \times K^{\times}$.

Proof - Let $C$ be a Carter subgroup of $G$, and let $B$ be a Borel subgroup containing $C$ such that either $U_{q}(B)$ is non-trivial for a prime $q$, or the integer $r=\bar{r}_{0}(B)$ is maximal for any such Borel subgroup. By Lemma 3.10, $B$ is nonnilpotent and we have $B \neq C$. Since $W(G)$ is non-trivial, Proposition 3.2 gives 
$N_{G}(C) \neq C$, and Fact $2.18(5)$ shows that $B$ does not contain $N_{G}(C)$. Hence by Theorem 3.12, there exists $w \in N_{G}(C) \backslash N_{G}(B)$. In particular, $C \leq B \cap B^{w}$ is abelian by Fact $2.22(3)$.

If we have $U_{q}(B) \neq 1$ for a prime $q$, then $B$ has a $G$-minimal subgroup $A$ of exponent q. If $C_{C}\left(A, A^{w}\right)$ is non-trivial, then $C_{G}\left(C_{C}\left(A, A^{w}\right)\right)^{\circ}$ is a proper connected definable subgroup of $G$ containing $A$ and $A^{w}$. Hence Fact $2.22(1)$ shows that $B$ (resp. $\left.B^{w}\right)$ is the unique Borel subgroup of $G$ containing $C_{G}\left(C_{C}\left(A, A^{w}\right)\right)^{\circ}$. This contradicts $B \neq B^{w}$. Consequently $C_{C}\left(A, A^{w}\right)$ is trivial, and $C$ is definably isomorphic to a subgroup of $B / C_{B}(A) \times B^{w} / C_{B^{w}}\left(A^{w}\right) \simeq B / C_{B}(A) \times B / C_{B}(A)$. If $B / C_{B}(A)=1$, then $C$ is trivial, contradicting that $C$ is a Carter subgroup of $G$. So, by Fact 2.8 , there is an interpretable field $K$ of characteristic $q$ such that $B / C_{B}(A)$ is definably isomorphic to a subgroup of $K^{\times}$. Hence we may assume that $U_{q}(B)=1$ for each prime $q$, so $r>0$.

We show that $B$ has a $B$-minimal homogeneous $U_{0, r}$-subgroup. By Fact $2.20(1)$, $U_{0}(B)$ is nilpotent, and by Fact $2.21\left[B, U_{0}(B)\right]$ is a homogeneous $U_{0, r}$-subgroup of $B$, so we may assume $\left[B, U_{0}(B)\right]=1$. Then $U_{0}(B)$ is central in $B$, and $C$ contains $U_{0}(B)$, so we have $U_{0}(C)=U_{0}(B) \leq Z(B)$. Thus we obtain

$$
U_{0}\left(B^{w}\right)=U_{0}(B)^{w}=U_{0}(C)^{w}=U_{0}(C)=U_{0}(B),
$$

and $U_{0}(B)$ is central in $\left\langle B, B^{w}\right\rangle=G$, contradicting the simplicity of $G$. Hence $B$ has a $B$-minimal homogeneous $U_{0, r}$-subgroup $A$.

If $C_{C}\left(A, A^{w}\right)=1$, then $C$ is definably isomorphic to a subgroup of $B / C_{B}(A) \times$ $B^{w} / C_{B^{w}}\left(A^{w}\right) \simeq B / C_{B}(A) \times B / C_{B}(A)$. If $B / C_{B}(A)=1$, then $C=1$, which contradicts that $C$ is a Carter subgroup of $G$. So, by Fact 2.8, there is an interpretable field $K$ such that $B / C_{B}(A)$ is definably isomorphic to a subgroup of $K^{\times}$. Hence we may assume that $C_{C}\left(A, A^{w}\right)$ is non-trivial.

Let $B_{0}$ be a Borel subgroup of $G$ containing $N_{G}\left(C_{C}\left(A, A^{w}\right)\right)^{\circ}$. Then $B_{0}$ contains $C, A$ and $A^{w}$. Since $B \neq B^{w}$, we have either $B_{0} \neq B$ or $B_{0} \neq B^{w}$. In the first case we consider $H_{0}=\left(B_{0} \cap B\right)^{\circ}$ and, in the second case, $H_{0}=\left(B_{0} \cap B^{w}\right)^{\circ}$. In particular $H_{0}$ contains $C$, and either $A$ or $A^{w}$, so we have $\bar{r}_{0}\left(H_{0}\right)=r$.

Let $B_{1}$ and $B_{2}$ be two distinct Borel subgroups of $G$ containing $H_{0}$, such that $H=\left(B_{1} \cap B_{2}\right)^{\circ}$ is maximal among all the choices of distinct Borel subgroups $B_{1}$ and $B_{2}$. Since $B_{1}$ and $B_{2}$ contain $H_{0}$, they contain $C$, and they are generous in $G$. Since $\bar{r}_{0}\left(H_{0}\right)=r$, the maximality of $r$ yields $\bar{r}_{0}(H)=\bar{r}_{0}\left(B_{1}\right)=\bar{r}_{0}\left(B_{2}\right)=r$. Thus, by Fact $2.20(1), U_{0}(H) \leq F\left(B_{1}\right) \cap F\left(B_{2}\right)$. In particular, this intersection is non-trivial. But Fact $2.22(2)$ implies that $F\left(B_{1}\right) \cap F\left(B_{2}\right)=1$, a contradiction.

\section{A Classification theOREM}

In this section we apply a particular case of Theorem 3.12 to a concrete classification problem and achieve a conceptual streamlining in the proof of a well-known theorem in the theory of simple groups of finite Morley rank. We will provide a new proof of one of the main ingredients of the analysis of strongly embedded subgroups in [BCJ07]:

Theorem 6.1. - If $G$ is a connected minimal simple group of odd type and of Prüfer 2-rank at least two, then $G$ has no strongly embedded subgroup.

Our new proof is direct and simple. It can also be expected that the main lines of the argument presented below will be generalized to other concrete problems in the analysis of simple groups of odd type. 
The notion of strong embedding was imported from finite group theory, and turned out to be almost as effective a tool as in its homeland. In order to appreciate its importance, it suffices to consult Section 10.5 of [BN94], [ABC08] or [BCJ07]. We will be content with saying that a simple group of finite Morley rank with a strongly embedded subgroup is conjectured to be isomorphic to $\mathrm{PSL}_{2}(K)$ where $K$ is algebraically closed of characteristic 2 , and the strongly embedded subgroups are the Borel subgroups. The following is one of the many equivalent definitions:

Definition 6.2. - Let $G$ be a group of finite Morley rank with a proper definable subgroup $M$. Then $M$ is said to be strongly embedded in $G$ if $I(M) \neq \emptyset$ and for any $g \in G \backslash M, I\left(M \cap M^{g}\right)=\emptyset$, where $I(X)$ denotes the set of involutions in $X$.

Note that it follows from the definition that a strongly embedded subgroup is self-normalizing. The presence of a strongly embedded subgroup imposes strong limitations on the structure of a group of finite Morley rank the most decisive of which concern involutions:

Fact 6.3. - $[\mathrm{BN} 94, \S 10.5]$ Let $G$ be a group of finite Morley rank with a strongly embedded subgroup $M$. Then the following hold:

(1) $N_{G}(S) \leq M$ for any non-trivial 2-subgroup $S$ of $M$.

(2) A Sylow 2-subgroup of $M$ is a Sylow 2-subgroup of $G$.

(3) The set $I(G)$ is a single conjugacy class in $G$; the set $I(M)$ is a single conjugacy class in $M$.

We will need from [BCJ07] the following three ingredients. The first two are general lemmas proven early in that article while the last one is the theorem that constitutes its "Case I".

Fact 6.4. - [BCJ07] Let $G$ be a minimal connected simple groups of finite Morley rank and odd type. Suppose that $G$ has Prüfer 2-rank at least two, and that $M$ is a strongly embedded subgroup of $G$. Then the following hold:

(1) [Lemma 2.3] $M^{\circ}$ is a Borel subgroup of $G$.

(2) [Lemma 2.6] $M^{\circ}$ is generous in $G$.

(3) [§4] No involution of $M^{\circ}$ lies inside $Z\left(M^{\circ}\right)$.

Apart from this important ingredient, our proof will be only using [BD09]. This use necessitates a certain care as we will try to avoid any reference to [Del08] since such a reference will potentially involve an implicit use of [BCJ07] and thus, a vicious circle. This special care is the reason why we verify towards the end of the proof that the Weyl group is of odd order. As was explained before Fact 3.11, in this particular case Theorem 3.12 has a proof using the relevant parts of [BD09] that do not use [Del08].

The rest of the ingredients are of a more general nature around genericity arguments such as Fact 2.14.

Proof of Theorem 6.1 - By way of contradiction, let us suppose that $G$ contains a strongly embedded subgroup $M$. By Fact $6.4(1)$ and (2), $M^{\circ}$ is a generous Borel subgroup of $G$.

By Fact 2.14, every 2-element in a group of odd type is contained in the connected component of its centralizer. It then follows from Fact $6.3(1)$ that $M^{\circ}$ contains all the 2-elements of $M$. Thus $M / M^{\circ}$ is of odd order. 
Next, we prove that $W(G)$ is of odd order. Let $C$ be a Carter subgroup of $M^{\circ}$. By Lemma 3.9, $C$ is also a Carter subgroup of $G$, and Facts 2.18 (2) and (4) show that $C$ contains a maximal 2-torus of $G$. Thus, the Sylow 2 -subgroup $T$ of $C$ is nontrivial, and since $T$ is characteristic in $C$, Fact 6.3 (1) implies that $M$ contains $N_{G}(C)$. Since $C$ is self-normalizing in $M^{\circ}$ by Fact $2.18(5)$, the quotient $N_{G}(C) / C$ is isomorphic to a subgroup of $M / M^{\circ}$, which is of odd order by the previous paragraph. It follows using Proposition 3.2 that $W(G)$ is of odd order.

We show that $M^{\circ}$ is not nilpotent and that $M$ is not connected. Since $G$ has odd type, $I\left(Z\left(F\left(M^{\circ}\right)\right)\right)$ is finite, so it is central in $M^{\circ}$, and Fact $6.4(3)$ forces $I\left(Z\left(F\left(M^{\circ}\right)\right)\right)=\emptyset$. Since $F\left(M^{\circ}\right)$ is nilpotent, this implies $I\left(F\left(M^{\circ}\right)\right)=\emptyset$, and $M^{\circ}$ is not nilpotent since $M / M^{\circ}$ is of odd order. Now, if $M$ is connected, then $M / F(M)$ is abelian (Fact 2.11), and all the involutions of $M$ have the same image in $M / F(M)$ by Fact $6.3(3)$. But $I(F(M))=\emptyset$, so a Sylow 2-subgroup of $M$ has just one involution. This contradicts Fact 6.3 (2) since $G$ has Prüfer 2-rank at least two, hence $M$ is not connected.

At this stage, Theorem 3.12 yields a contradiction. As $W(G)$ has odd order, the remarks preceding Fact 3.11 show that we have avoided applications of [Del08] and hence of [BCJ07] as well.

\section{Acknowledgements}

This paper was initially part of a larger one, a presentation that we have modified following the careful and frank report of an earlier referee. The final version of the second presentation has much benefited from similar qualities of the second refereeing. The authors thank both referees.

\section{REFERENCES}

[AB09] Tuna Altinel and Jeffrey Burdges. On analogies between algebraic groups and groups of finite Morley rank. J. London Math. Soc., (2) 78, no. 1:213-232, 2008.

[ABC08] T. Altınel, A. V. Borovik and G. Cherlin. Simple Groups of Finite Morley Rank. Mathematical Surveys and Monographs 145, 2008. Providence, RI: American Mathematical Society (AMS).

[AC04] Tuna Altinel and Gregory Cherlin. Simple $L^{*}$-groups of even type with strongly embedded subgroups. J. Algebra, 272:95-127, 2004.

[BBC07] Alexandre Borovik, Jeffrey Burdges, and Gregory Cherlin. Involutions in groups of finite Morley rank of degenerate type. Selecta Math., 13(1):1-22, 2007.

[BN94] Alexandre Borovik and Ali Nesin. Groups of Finite Morley Rank. The Clarendon Press Oxford University Press, New York, 1994. Oxford Science Publications.

[Bur04] Jeffrey Burdges. A signalizer functor theorem for groups of finite Morley rank. $J$. Algebra, 274:215-229, 2004.

[Bur06] Jeffrey Burdges. Sylow theory for $p=0$ in solvable groups of finite Morley rank. $J$. Group Theory, 9:467-481, 2006.

[Bur07] Jeffrey Burdges. The Bender method in groups of finite Morley rank. J. Algebra, 312(1):33-55, 2007.

[BC08b] Jeffrey Burdges and Gregory Cherlin. Semisimple torsion in groups of finite morley rank. J. Math. Logic, 9, No. 2, 183-200, 2009.

[BCJ07] Jeffrey Burdges, Gregory Cherlin, and Eric Jaligot. Minimal connected simple groups of finite Morley rank with strongly embedded subgroups. J. Algebra, 314(2):581-612, 2007.

[BD09] Jeffrey Burdges and Adrien Deloro. Weyl groups of small groups of finite Morley rank. Israel J. Math., 179:403-423, 2010.

[Che05] Gregory Cherlin. Good tori in groups of finite Morley rank. J. Group Theory, 8:613$621,2005$. 
[CJ04] G. Cherlin and E. Jaligot. Tame minimal simple groups of finite Morley rank. J. Algebra, 276, 13-79, 2004.

[Del08] Adrien Deloro. Groupes simples connexes minimaux non-algébriques de type impair. J. Algebra, 319:1636-1684, 2008.

[Fré00a] Olivier Frécon. Sous-groupes anormaux dans les groupes de rang de Morley fini résolubles. J. Algebra, 229(1):118-152, 2000.

[Fré06a] Olivier Frécon. Around unipotence in groups of finite Morley rank. J. Group Theory, 9:341-359, 2006

[Fré06b] Olivier Frécon. Carter subgroups in tame groups of finite Morley rank. J. Group Theory, 9:361-367, 2006.

[Fré08] Olivier Frécon. Conjugacy of Carter subgroups in groups of finite Morley rank. $J$. Math Logic. J. Math. Logic, 8, No. 1, 41-92, 2008.

[Fré09] Olivier Frécon. Pseudo-tori and subtame groups of finite Morley rank. J. Group Theory, 12:305-315, 2009 .

[FJ05] Olivier Frécon et Éric Jaligot. The existence of Carter subgroups in groups of finite Morley rank. J. Group Theory, 8:623-633, 2005.

[FJ08] Olivier Frécon et Éric Jaligot. Conjugacy in groups of finite Morley rank. Model Theory with Applications to Algebra and Analysis Volume 2, pp. 1-58, London Math. Soc. Lecture Note Ser., 350, Cambridge Univ. Press, Cambridge, 2008

[Gor68] D. Gorenstein. Finite groups. Harper \& Row, Publishers, 1968.

[Hum81] J.E. Humphreys. Linear algebraic groups. Springer-Verlag, 2nd edition, 1981.

[Jal01] Éric Jaligot. Full Frobenius groups of finite Morley rank and the Feit-Thompson theorem. Bull. Symbolic Logic, 7(3):315-328, 2001.

[Jal06] Éric Jaligot. Generix never gives up. J. Symbolic Logic 71:599-610, 2006.

[Mac70Gr] Angus Macintyre. On $\omega_{1}$-categorical theories of abelian groups. Fund. Math. 70 (3):253-270, 1970.

[Mac71Fi] Angus Macintyre. On $\omega_{1}$-categorical theories of fields. Fund. Math. 71 (1):1-25, 1971.

[Nes91] Ali Nesin. Poly-separated and $\omega$-stable nilpotent groups. J. Symbolic Logic 56:694699, 1991.

[Rei] J. Reineke. Minimale gruppen. Z. Math. Logik Grundl. Math. 21:357-379, 1975.

[Wag94] F.O. Wagner. Nilpotent complements and Carter subgroups in stable $\Re$-groups. Arch. Math. Logic 33(1):23-34, 1994.

Université Lyon 1, Institut Camille Jordan, 43 Blvd du 11 novembre 1918, 69622 Villeurbanne Cedex, France

E-mail address: altinel@math.univ-lyon1.fr

Université Lyon 1, Institut Camille Jordan, 43 Blvd du 11 novembre 1918, 69622 Villeurbanne Cedex, France

E-mail address: burdges@math.univ-lyon1.fr

Laboratoire de Mathématiques et Applications, Université de Poitiers, Téléport 2 - BP 30179, Boulevard Marie et Pierre Curie, 86962 Futuroscope Chasseneuil Cedex, FRANCE

E-mail address: olivier.frecon@math.univ-poitiers.fr 